\title{
¿JUSTICIA EN LOS BALCANES? EL FALLO DE LA CORTE INTERNACIONAL DE JUSTICIA SOBRE EL GENOCIDIO EN BOSNIA
}

\author{
Juan Manuel PORTILLA GÓMEZ* \\ SUMARIO: I. Marco histórico-referencial. II. Análisis del fallo \\ de la Corte. III. Reflexiones finales.
}

\section{MARCO HISTÓRICO-REFERENCIAL}

\section{Cambio y conflicto en la antigua Yugoslavia}

Los conflictos bélicos han estado presentes por largo tiempo en la región de los Balcanes, y el territorio de la antigua Yugoslavia ha sido teatro, en diversos momentos de su historia, de cruentos enfrentamientos con terribles consecuencias. ${ }^{1}$ El incidente que detonó la Primera Guerra Mundial tuvo su origen en Sarajevo, el 28 de junio de 1914, cuando un nacionalista serbio asesinó al archiduque Francisco Fernando. Después de la guerra se unieron diversos territorios eslavos para formar Yugoslavia, con su capital en Belgrado y dominada por los serbios. Al comienzo de la Segunda Guerra Mundial, Yugoslavia se encontraba inmersa en una guerra civil y

* Doctor en derecho internacional. Profesor en la Facultad de Estudios Superiores Acatlán de la UNAM.

1 Bosnia fue parte del imperio turco-otomano hasta 1878 y luego su territorio quedó dentro del imperio austro-húngaro hasta la Primera Guerra Mundial. Así describe Kissinger a la Bosnia de entonces: "This no man land between the Ottoman Habsburg Empires, which contained Roman Catholic, Orthodox, and Muslim religions, and Croatian, Serbian, and Muslims populations, had never been a state or even self-governing. It only seemed governable if none of theses groups was asked to submit to the others. For forty years, Bosnia Herzegovina had been under Turkish suzerainty, Austrian administration, and local autonomy without experiencing a serious challenge to this multinational arrangement which left the issue of ultimate sovereignty unsettled. Kissinger A., Henry, Diplomacy, Nueva York, Simon \& Schuster, 1994, p. 195. 
al finalizar ésta, se formó, bajo el liderazgo del comunista Josip Bros Tito, un Estado denominado República Federal Socialista de Yugoslavia. Al fallecer éste, en 1980, la Federación se desquebrajó y se dio un violento rebrote de los nacionalismos extremos. En 1987 asumió el poder el serbio de origen montenegrino Slobodan Milosevic, partidario de una Gran Serbia y promotor del nacionalismo serbio a ultranza. ${ }^{2}$ En lo años siguientes, las repúblicas buscaron su emancipación, declarando Croacia y Eslovenia su independencia el 25 de junio de 1991, Macedonia el 17 el septiembre de 1991 y Bosnia-Herzegovina (en adelante Bosnia) hizo lo propio el 15 de octubre del mismo año. Luego de un inicial ataque a Eslovenia, el 27 de junio, y de una cruenta guerra de un año contra Croacia, Yugoslavia volcó su atención sobre Bosnia, visiblemente debilitada por el embargo de armas impuesto por la ONU. Hacia finales de 1993, los serbios, liderados por Radovan Karadzic, establecieron su propia Republika Srpska en el Este, y un ejército serbio bosnio comandado por Ratko Mladic controló casi tres cuartos del país; los croatas fueron prácticamente expulsados y los musulmanes fueron obligados a replegarse hacia los centros urbanos.

El conflicto atrajo a todas las facciones políticas y religiosas en la región, suscitándose actos de tortura, violación y genocidio. La Unión Europea trató de mediar, sin éxito, y la inicial participación de la ONU se limitó a suministrar ayuda humanitaria; posteriormente se envió una Fuerza de Protección de las Naciones Unidas (Unprofor), misma que estableció seis "áreas protegidas" principalmente para los musulmanes. ${ }^{3} \mathrm{La}$ devastación abarcó de junio de 1991 hasta diciembre de 1995.

El Acuerdo Marco General para la Paz en Bosnia-Herzegovina, comenzado en la base Whright Paterson de la Fuerza Área estadounidense, próxima a Dayton, Ohio, y firmado en París el 14 de diciembre de

2 Al mismo tiempo, en Zagreb surgía la figura de Franco Tudjman, presidente de Croacia y nacionalista extremo.

3 Todas y cada una de ellas cayeron en manos de los serbios, destacando Srebrenica, a cargo de un contingente holandés, que fue escenario en 1995 del genocidio reconocido por la Corte. A la Unprofor, le siguió la Fuerza de Estabilización (Sfor) de la OTAN, que después fue remplazada por la Fuerza de la Unión Europea (Eurofor). Véanse www.un.org/spanish/Depts/dpko/dpko/co_mission/orgfor.htm y www.nato.int/docu/update!2004/12-december/el202a.htm. 
1995 por Bosnia, Croacia y Yugoslavia, puso término oficialmente al conflicto en la antigua Yugoslavia. ${ }^{4}$

\section{Trayectoria del crimen de genocidio}

La penalización del genocidio, bajo el derecho internacional, proviene de los años inmediatos posteriores a la Segunda Guerra Mundial. ${ }^{5}$ En 1948, las Naciones Unidas adoptaron la Convención para la Prevención y la Sanción del Delito de Genocidio (en adelante la Convención de Genocidio). ${ }^{6}$

No obstante ello, la comisión del llamado "crimen de crímenes" no se detuvo, y los actos de genocidio volvieron a suscitarse en diversas latitudes y escenarios. Así, a los genocidios acaecidos en el siglo pasado en el curso de la primera y segunda guerras mundiales: el de Turquía contra los armenios, y de Alemania contra diversos grupos étnicos, respectivamente, vinieron a sumarse otros, en plena guerra fría, como los del Khemer Rouge de Pol Pot en Camboya, el de la Falange Cristiana Libanesa

4 Es importante subrayar que el arreglo pactado requirió de todas las partes el respeto a ciertas instrumentos internacionales, incluyendo la Convención de Genocidio. Véase Szaz, Paul C., "The Protection of Human Rights Through the Dayton-Paris Peace Agreement on Bosnia”, American Journal of International Law, núm. 90, Washington, 1996, p. 301. En este acuerdo, el nuevo Estado bosnio quedó conformado por la Federación croata-musulmana y por los serbio-bosnios, Republika Sprska, correspondiéndole a esta última el 51\% del territorio. El problema de esta solución es que, aunque existen tres pueblos en el territorio bosnio (musulmanes $40 \%$, serbios $30 \%$ y croatas $17 \%$ ), la Constitución sólo reconoce dos entidades. Los que censuran esta solución señalan que fue resultado del genocidio contra los bosnios musulmanes, antes prevista por un acuerdo entre Milosevic y Tudjman, facilitado por la legalización de la Republika Sprska, conseguida mediante presiones de Holbrook, el negociador estadounidense, sobre Izebigovic, presidente bosnio musulmán.

5 El término genocidio fue acuñado por el abogado judío polaco Raphael Lemkin. Véase Lemkin, Raphael, Axis Rule in Occupied Europe: Laws of Occupation, Analysis of Government, Proposals of Redress, Washington, Carnegie Endowment for World Peace, 1944.

6 Aprobada y abierta firma y ratificación, o adhesión, por la Asamblea General en su Resolución 260 A (III), del 9 de diciembre de 1948. De conformidad con el artículo XII, entró en vigor el 12 de enero de 1951. Antes de la Convención de Genocidio, este ilícito penal no se encontraba tipificado como tal en ningún instrumento internacional, y aún en el Estatuto del Tribunal de Nuremberg no se estableció como una categoría separada de crímenes contra la humanidad. Véase Cassese, Antonio, International Criminal Law, Oxford, Oxford University Press, 2003, p. 96. 
de Michael Aoun (en connivencia con Ariel Sharon, comandante de las tropas israelíes) en el campamento de refugiados palestinos de Sabra y Shatila. $^{7}$

En la llamada posguerra fría, una vez derrumbado el Muro de Berlín y desintegrada la Unión Soviética, la violación del derecho humanitario y de los derechos humanos volvió a hacerse patente en el desarrollo de terribles conflictos en que la exacerbación de las tensiones étnicas y ultranacionalistas, en combinación con los intereses (o desintereses) de las potencias, dio por resultado la reaparición del crimen de genocidio en la década de los noventa en los Balcanes, y enseguida en África. Para efectos del presente análisis, sólo nos ocuparemos del primero de los casos. $^{8}$

El genocidio cometido en Bosnia en contra de la población musulmana tiene dos vertientes: una que implica la responsabilidad del Estado, a través de sus órganos, en su calidad de sujeto de derecho internacional, y otra, que implica la responsabilidad de las personas a título individual. Este segundo rubro ha constituido la tarea del Tribunal Penal Internacional para la ex Yugoslavia desde su creación, en 1993, mediante resolución del Consejo de Seguridad de la ONU acorde con el capítulo VII de la Carta. ${ }^{9}$ Respecto al primer tipo de responsabilidad, es competencia de la

7 Es interesante observar la reticencia a calificar ciertas situaciones como genocidio, toda vez que tanto el Consejo de Seguridad como la Asamblea General de la ONU se refirieron sólo a una "masacre de civiles palestinos". Véanse resoluciones S/RES/521(1982) del 19 de septiembre de 1982 y A/RES/ES-7/9 del 24 de septiembre de 1982. Sin embargo, la Asamblea General reconoció posteriormente que se trató de "un acto de genocidio" en su resolución 37/123 D del 16 de diciembre de 1982, siendo esta la primera vez que un órgano de la ONU se refiriese al genocidio.

8 En África, para Rwanda y Sierra Leone se crearon tribunales de la ONU, y la Corte Penal Internacional se encuentra procesando los casos de la República Democrática del Congo, Uganda y Darfur, además de estar realizando una investigación en la República Centroafricana. Para otras regiones, se han creado tribunales "híbridos" en Kosovo, Timor Oriental y Cambodia. Véanse Schabas, William A., The UN International Criminal Tribunals. The Former Yugoslavia, Rwanda and Sierra Leone, Cambridge University Press, 2006 y Dickinson, Laura A., "The Promise of Hibrid Courts", American Jourrnal of International Law, Washington, American Society of International Law, vol 97, núm. 295, 2003. Asimismo, consúltese el sitio de la Corte Penal Internacional, www.icc-cpi.int.

9 Resolución 827 del 25 de mayo de 1993, adoptada por acuerdo general sin votación de los 15 miembros del Consejo de Seguridad, por la que se crea el Tribunal Internacional para la Persecución de Personas Responsables de Serias Violaciones al Derecho 
Corte Internacional de Justicia conocer de casos de Estados conforme a la Convención de Genocidio.

\section{La acción de Bosnia}

El 20 de marzo de 1993, Bosnia presentó su caso contra Yugoslavia ante la Corte Internacional de Justicia de la Haya (en adelante la Corte), alegando la violación de la Convención para la Prevención y la Sanción del Delito de Genocidio (en adelante, la Convención de Genocidio). ${ }^{10}$ Comenzaba así el juicio más largo en la historia de la Corte, mismo que habría de concluir el 26 de febrero del 2007, y que por vez primera ésta se pronunciase sobre el fondo de un asunto de genocidio. ${ }^{11}$ En el curso de los catorce años de procedimientos, la parte demandada cambió dos veces de nombre. Al principio se trató de la República de Yugoslavia; en el 2001 el nombre del país cambió a Serbia y Montenegro, para finalmente, en junio de 2006, tras la secesión de Montenegro, quedar sólo como Serbia. Así, la Corte en su decisión final descartó a esta nueva república como parte en el caso, aunque siguió considerando su responsabilidad sobre hechos pasados en su condición de parte en la Convención de Genocidio respecto a su obligación de cooperar para castigar a los perpetradores de genocidio (párrafos 71-78).

En su fase procesal, el juicio se basó en decisiones previas de la Corte en las que ésta encontró elementos para establecer su competencia. En el discernimiento sobre su jurisdicción, la Corte no estimó necesario considerar algunas cuestiones legales, planteadas por las partes en su inter-

Internacional Humanitario cometidas en el Territorio de la ex Yugoslavia desde 1971 (ICTY).

10 Ese mismo año, en respuesta a una solicitud bosnia, la Corte dictó medidas provisionales, prohibiendo potenciales violaciones a la Convención de Genocidio.

11 Además del caso que nos ocupa, ha habido otros cuatro relativos a genocidios sometidos a la Corte, de conformidad con el artículo 9o. de la Convención de Genocidio, a saber los casos: prisioneros pakistaníes (Pakistán vs. India), y legalidad del uso de la fuerza (Yugoslavia vs. Miembros de la OTAN) y Croacia vs. Yugoslavia. El primer caso (1973) fue retirado de la Corte a petición de Pakistán; en el segundo, la Corte (1999) desestimó su jurisdicción, y el tercero (1999) aún no llega a la fase del fondo. Asimismo, la Corte emitió una opinión consultiva (1951) sobre Reservas a la Convención para la Prevención y la Sanción del Delito de Genocidio. Véase, Schabas, William A., Genocide in International Law: The Crime of Crimes, Cambridge University Press, 2000, pp. 424 y 423. 
cambio de sus posicionamientos escritos y a través de sus intervenciones orales. Así, el 11 de julio de 1996, la Corte se pronunció afirmativamente sobre la admisibilidad y jurisdicción de la acción incoada por Bosnia; rechazando las objeciones preliminares de Serbia, así como otras bases de jurisdicción planteadas por Bosnia que no fueran el artículo IX de la Convención de Genocidio. ${ }^{12}$

En nuestro estudio del fondo del asunto, seguiremos la ruta temática desarrollada por la Corte, sin considerar las opiniones disidentes e individuales, comenzando por una sinopsis y continuando con un formato de preguntas y respuestas sobre las cuestiones más importantes del fallo sobre las que evaluaremos la pertinencia de la metodología y el enfoque utilizados.

\section{ANÁLISIS DEL FALLO DE LA CORTE ${ }^{13}$}

\section{Sinopsis del fallo}

Siguiendo los pasos del Tribunal Penal Internacional para la ex $\mathrm{Yu}-$ goslavia en sus conclusiones sobre los hechos, y aunque los crímenes contra la humanidad estén fuera de su competencia, la Corte estableció que Serbia cometió crímenes contra la humanidad a gran escala. Coincidió con dicho tribunal en que la masacre de Srebrenica fue un genocidio cometido por los serbio-bosnios. Pero la Corte señaló que para que un Estado sea responsable de Genocidio, es necesario que el crimen haya

12 Luego del fallo sobre competencia y admisibilidad, ocurrió una serie de incidencias que demoraron sustancialmente el juicio. Por una parte, en el 2000 la Republika Srpska intentó retirar el caso, y por la otra, a principios del 2001, Serbia solicitó la suspensión del proceso, pues alegó que su admisión en noviembre del 2001 como miembro de las Naciones Unidas cambiaba su posición, en la medida que careció de tal calidad entre 1992 a 2000. Para mayores detalles de la historia procesal del caso, véanse Milanovic, Marko, "State Responsability for Genocide", European Journal of International Law, vol. 17, núm. 3, 2006, pp. 590-592, así como Morgan-Foster, Jason y Oliver-Savoie, Pierre, "World Court finds Serbia Responsible for Breaches of Genocide Convention, but not Liable for Commiting Genocide", ASIL Insight, 3 de abril, 2007, vol. 11, issue 9, www.asil.org.

13 Fallo de la Corte Internacional de Justicia sobre el caso Application of the Convention on the Prevention and Punishment of the Crime of Genocida (Bosnia and Herzegovina $v$. Serbia and Montenegro), Lista general 91, emitido el 26 de febrero del 2007, véase $w w w$.icj-cij/docket/index. 
sido cometido por órganos de ese Estado, o bajo instrucciones, directrices o control efectivo de los mismos. La Corte admitió que en los primeros años del conflicto existió tal vínculo entre Belgrado y los mandos serbio-bosnios, excepto en 1995 cuando ocurrió la caída de Srebrenica. Por ello, no puede concluir, sobre la única base de las pautas de violencia suscitadas, que Serbia haya tenido la intención de aniquilar, parcial o totalmente, a la población musulmana de Bosnia como lo requiere la Convención de Genocidio. Por lo tanto, Serbia es considerada no responsable del genocidio cometido en Srebrenica y, consecuentemente, tampoco responsable de complicidad por dicho crimen.

"La complicidad no es un término que exista en la terminología actual de la responsabilidad internacional", explicó la Corte, pero es un término utilizado por la Convención de Genocidio. La Corte dijo que para que se establezca la complicidad de un Estado o de sus órganos, éste debe suministrar "ayuda o asistencia". Aún así, debe probarse "un vínculo entre la intención específica de los autores del crimen y el móvil que inspira la acción del cómplice". Asimismo, la Corte dijo que "sin duda alguna, la ayuda sustancial, de naturaleza política, militar y financiera suministrada por la RFY (República Federal de Yugoslavia) a la República Sprska y al VRS (Ejército de la Republica Sprska), mucho antes de los acontecimientos de Srebrenica, continuó en el curso de los acontecimientos. Sin embargo, la Corte encontró que no pudo establecerse fuera de toda duda razonable, que las autoridades de la RFY "estuvieran claramente concientes de que un genocidio estaba por llevarse a cabo o en proceso de cometerse". Según la Corte, la decisión de destruir a los musulmanes de Srebrenica, "fue tomada por los serbio-bosnios poco antes de su ejecución, empresa que requirió de un lapso muy reducido (básicamente entre el 13 y el 16 de julio), no obstante el excepcionalmente elevado número de víctimas. La RFY en efecto proveyó ayuda y asistencia, pero sin el "pleno conocimiento" de que esta ayuda sería utilizada para cometer genocidio.

El primer artículo de la Convención de Genocidio expresa la obligación de los Estados de "prevenir y sancionar el genocidio". La Corte consideró responsable a Serbia de la violación continua de dicha convención por la no entrega del general serbio-bosnio Ratko Mladic al ICTY, presumiblemente oculto en Serbia. Pero de mayor importancia son las conclusiones de la Corte sobre el hecho de no haber impedido el genocidio. Para establecer tal falta, la Corte dijo que era necesaria una "evalua- 
ción concreta", concluyendo que la RFY podía y debía haber prevenido el genocidio en Srebrenica. Para la Corte fueron factores determinantes la distancia geográfica, la fuerza de los vínculos políticos, y otros más, así como la influencia práctica y jurídica. A partir de que un Estado se percate, o debía normalmente darse cuenta, de la existencia de un serio riesgo de que se cometa genocidio, si dicho Estado dispone de medios disuasivos, tiene la obligación de utilizarlos en la medida que la ocasión lo permita.

Una línea muy delgada separa la complicidad de la falta de prevención. De acuerdo con la Corte, la complicidad es un acto cometido a sabiendas de la intención del autor del crimen, mientras que la falta de prevención de un crimen es una omisión sustentada por el desconocimiento del grave peligro de que los actos de genocidio puedan ser cometidos por el otro Estado. La Corte precisó que los dirigentes en Belgrado, "y en primer lugar el presidente Milosevic", sabían del profundo odio de los serbio-bosnios contra los musulmanes de Srebrenica y tenían más influencia en la región que cualquier otro Estado.

\section{2. ¿Se presentó alguna novedad sobre la jurisdicción de la Corte?}

En el fallo del 26 de febrero del 2007 sobre el fondo de la controversia, no hubo ningún cambio respecto a la autoridad de la Corte para conocer del caso, y ésta sólo confirmó su previa posición en este rubro. Las primeras 53 páginas de la decisión están dedicadas al recuento del caso y a sustentar su jurisdicción sobre este asunto.

No es sino hasta la página 54 que comenzó la discusión sobre la aplicabilidad de la Convención de Genocidio. Ahí la Corte confirmó su jurisdicción para conocer sobre controversias bajo la Convención de Genocidio, limitando nuevamente su alcance sólo al ámbito del artículo IX de la Convención. ${ }^{14}$ El efecto de esto es claramente expresado en los siguientes términos: "The Court has no power to rule on alleged breaches of ot-

14 Artículo IX. Las controversias entre las partes contratantes, relativas a la interpretación, aplicación o ejecución de la presente Convención, incluso las relativas a la responsabilidad de un Estado en materia de genocidio o en materia de los otros actos enumerados en el artículo III, serán sometidas a la Corte Internacional de Justicia a petición de una de las partes en la controversia. Si bien la redacción del artículo es clara, los trabajos preparatorios evidencian ambigüedades y apreciaciones confusas; para conocer el debate suscitado en torno a este artículo, véase Schabas, op. cit., nota 11, pp. 421-426. 
her obligations under international law, not amounting to genocide, particularly those protecting human rights in armed conflict" (párrafo 147). ${ }^{15}$

Lo anterior significa que se buscó concentrar todo el peso del fallo sobre la cuestión de que los acontecimientos referidos constituyesen o no genocidio, ya que la Corte explícitamente descartó pronunciarse sobre la comisión de otros ilícitos como crímenes contra la humanidad o crímenes de guerra. De ello se deriva la exclusión del veredicto de todo conjunto de hechos que implicara cualquier otro acto distinto al genocidio. Sin embargo, en distintas consideraciones de la Corte pareciera haber una marcada implicación de que los hechos corresponden a la definición de crímenes contra la humanidad, aunque el párrafo arriba citado excluye determinantemente tal posibilidad. Al respecto, conviene señalar que si bien los crímenes contra la humanidad y el genocidio comparten diversos rasgos, cada uno presenta peculiaridades propias que los hace diferentes. ${ }^{16}$

15 Al transcribir párrafos completos del fallo de la Corte, hemos optado por no traducirlos al español. La versión elegida ha sido la inglesa, debido a que además de ser la más difundida, constituye el texto auténtico. Asimismo, hemos dejado las citas textuales de autores extranjeros en su lengua original.

16 Antonio Cassese explica la relación entre ambos de la siguiente manera: "Both categories share at least three elements: i) they encompass very serious offences that shock our sense of humanity in what they constitute attacks on the most fundamental aspects of human dignity; ii) they do not constitute isolated events but are instead normally part of larger context, either because they are large-scale and massive infringements of human dignity or because they are linked to a broader practice of misconduct; iiii) although they need not be perpetrated by State officials or by officials of entities such as insurgents, they are usually carried out with the complicity, connivance, or at least the toleration or acquiescence of the authorities./ However, the objective and subjective elements of the two crimes differ in many respects. As for the objective element, the two crimes may undoubtedly overlap to some extent: for instance: killing members of an ethnic or religious group may as such fall under both categories; the same holds true for causing serious bodily or mental harm to members of a racial or religious group, or even for the other classes of protected group. However, crimes against humanity have a broader scope, for they may encompass acts that do not come within the purview of genocide (for instance, imprisonment and torture). By the same token, there may be acts of genocide that normally... are not held to fall within the other category of crime (for instance, killing detained military personnel belonging to a particular religious or racial group, by reason of their membership of that group). Thus, from the view point of their objective elements, the two categories are normally "reciprocally special", in that they form overlapping circles which nevertheless intersect only tangentially. By contrast, from the pers- 


\section{3. ¿Puede un Estado ser acusado de genocidio?}

El siguiente gran rubro que abordó la Corte es si el genocidio puede considerarse legalmente como una cuestión de responsabilidad estatal, independientemente de la responsabilidad individual de quienes lo cometen. La posición de los representantes serbios fue de que: "The Genocide Convention does not provide for the responsibility of States for acts of genocide as such. The duties prescribed by the Convention relate to the prevention and punishment of the crime of genocide when the crime is committed by individuals" (párrafo 156).

La Corte concluyó que la Convención no excluye a los Estados de la comisión de genocidio, y que los Estados pueden también ser responsables de complicidad (párrafos 166-169). Lo que es más importante aquí es la afirmación de la Corte sobre la "dualidad de la responsabilidad" (párrafo 173), por la cual el derecho internacional obliga tanto a individuos como a Estados. Así la Corte determinó que: "If an organ of the State, or a person or group whose acts are legally attributable to the State, commits any of the acts proscribed by Article III of the Convention, the international responsibility of that State is incurred" (párrafo 179).

La Corte determinó que un Estado ha cometido genocidio aún sin tener a ningún individuo convicto por dicho crimen (párrafos 180-182). La implicación más fuerte de esto se relaciona con los sujetos indiciados por genocidio en el Tribunal Penal Internacional para la ex Yugoslavia. Al respecto, la Corte agregó que no tenía que pronunciarse sobre la culpabilidad o inocencia de los mismos, ya que se encontraba limitada a determinar si sus "actos son legalmente atribuibles al Estado".

El principal obstáculo enfrentado por la Corte lo encontramos dentro del propio sentido de la Convención de Genocidio, por cuanto ésta se enfoca principalmente hacia los individuos, y no obstante imponer obligaciones a los Estados, no dispone expresamente que éstos puedan ser culpables de genocidio. Sin embargo, es difícil pensar que los individuos puedan operar por sí mismos con recursos y mecanismos sin el respaldo del aparato público. ${ }^{17}$

pective of the mens rea of both offences, the two categories do not overlap al all". Cassese, op. cit., nota 6, p. 106.

17 Al plantearse esta idea en el sentido opuesto respecto a si un Estado puede cometer un crimen, Milanovic responde que sí y no: “A state can do nothing by itself —it can 


\section{4. ¿Aplicó la Corte un concepto demasiado restringido de genocidio?}

Es claro que la Corte optó por un concepto muy restringido de genocidio y por una evidencia con un estándar excesivo para demostrar los elementos del crimen. Los términos más complicados de la Convención de Genocidio son: "intención", "grupo" y "parte". Veamos cada uno.

La cuestión de la intención es, probablemente, el mayor foco de controversia en el caso. La Convención de Genocidio requiere la demostración de intención genocida, e insiste en que debe ser "definida muy precisamente" (párrafo 189). La Corte no estimó convincente la evidencia de intención presentada por los demandantes (párrafos 371-372 y 374-376). Esto significa que no bastó el hecho de que los crímenes hayan tenido lugar, ni que haya habido una pauta de acontecimientos en ese sentido. Una determinación de genocidio, al contrario de una determinación de crímenes contra la humanidad, requiere de una evidencia específica y directa de intención (párrafo 188). Pero como ya vimos, la Corte ya había determinado (párrafo 147) que no tiene autoridad para determinar la comisión de crímenes contra la humanidad.

De manera similar, la Corte decidió que el término "limpieza étnica" carece de significado legal específico, y determinó que en ausencia de evidencia específica de intención: "Neither the intent as a matter of policy, to render an area ethnically homogenous, nor the operations that may be carried out to implement such policy, can as such be designated as genocide" (párrafo 190).

Esto pareciera, nada menos, que una reducción al absurdo por parte de la Corte ya que dicho razonamiento descartó el uso de la intención como evidencia de intención. Otra cuestión que adolece de ambigüedad es la contenida en el artículo II de la Convención de Genocidio respecto a la intención de destruir, todo o en parte, un grupo nacional, étnico, racial o religioso, como tal. Los elementos controversiales tienen que ver con definir "grupo" y "parte". La Corte se basó esencialmente en la jurisprudencia del ICTY y el ICTR para llegar a una definición absolutamente estrecha de "grupo", mismo que debe tener una identificación positiva

only act through individuals, who would in the overwhelming majority of cases be its de jure organs- But just as individuals can commit international crimes when acting in their official capacity, so can their criminal acts be attributed to a state. Yet, this state responsibility for an international crime is by its nature not criminal, but remains "civil'". Milanovic, op. cit., nota 12, p. 562. 
(párrafo 194); lo cual pareciera confirmar la objeción de los demandados de que la categoría de "no serbios" es demasiado vaga como para constituir un "grupo. ${ }^{18} \mathrm{Al}$ mismo tiempo, utilizaron un peculiar criterio interpretativo para establecer estándares en la definición de "parte"; la "parte" debe ser "sustancial" pero puede ser también "limitada geográficamente" (párrafos 198-200). Esto le vino muy bien a la Corte, pues le permitió determinar que el genocidio solamente ocurrió en lugar específico (digamos, Srebrenica) sin tener que establecer que dicho crimen fue cometido en todo el país.

\section{5. ¿Adoptó la Corte un estándar excesivamente alto de evidencia?}

En otra decisión que tendrá fuertes implicaciones en casos futuros, la Corte encontró que el criterio de prueba debe ser alto por cuanto las acciones en contra de un Estado que impliquen cargos de excepcional gravedad deben probarse por evidencia que sea totalmente conclusiva (párrafo 209). Así que rechazó la pretensión de que los cargos podrían probarse mediante una pauta de actos que habla por sí misma (párrafo 207); esta determinación implicó el que no fuera posible establecer el $d o-$ lus specialis o special intent en la comisión de un conjunto de atrocidades cometidas en muchas comunidades durante un largo tiempo. ${ }^{19} \mathrm{Se}$

18 Estamos de acuerdo con Milanovic en que el genocidio no es simplemente un crimen en contra de los individuos, debido a su pertenencia en un grupo, sino un ataque a la existencia de grupos humanos protegidos, y en que la lista de éstos es también exhaustiva - la destrucción de otros grupos políticos o sociales-. Al detallar sobre la problemática de los grupos protegidos señala: "Even if the list of protected groups is exhaustive, it still needs to be determined how to define a national, ethnic, racial or religious group. In most cases this is relatively easy. In the case of Rwanda, however, the ICTR had to establish whether the Tutsi could be subsumed under one of these categories, and encountered great difficulties arising from the relatively artificial distinction made between the Tutsi and the Hutu. The ICTR therefore oscillated from an objective approach (i.e., whether ethnic or racial groups, for instance, are defined by certain objective characteristics), to more subjective (or more anthropological) approaches (i. e. how the group identifies itself, or how the perpetrator identifies the group)". See, e. g., Prosecutor $v$. Rutaganda, ICTR-96-3, Trial Chamber judgment of 6 de diciembre de 1999: Appeals Chamber judgement of 26 de mayo de 2003, ICTR judgments can be found at www.ictr.org. Milanovic, op. cit., nota 12, p. 557.

19 Coincidimos con Schabas al señalar que la expresión dolus specialis es propia del sistema penal continental, mientras que el término special intention corresponde al Common Law. Schabas, op. cit., nota 8, pp. 164 y 165. Por su parte, Cassese refiere a la spe- 
decidió tratar con excesiva cautela los materiales de evidencia especialmente preparados para el caso y los provenientes de una sola fuente (párrafo 213), mientras que las decisiones del ICTY se consideraron confiables (más no las acusaciones), en virtud de que habían sido probadas tanto por procedimientos internos de fiscalía y defensa, como por interrogatorios rigurosos (párrafo 214). También dieron peso a informes de órganos oficiales o independientes derivados de procesos judiciales o quasi-judiciales (párrafo 227). La última determinación se refiere específicamente al informe de 1999 del secretario general de la ONU sobre la caída de Srebrenica.

Un problema recurrente en la configuración del requerimiento de intención conforme a la Convención de Genocidio es si debe exigirse la exhibición de un documento en el cual se demuestre claramente la intención genocida. En este sentido, no hay noticia de que tal documento exista en algún caso registrado de genocidio, y las razones por las que un gobierno podría tener para no producir tal documento son demasiado obvias.

Al respecto, Cassese censuró acremente la decisión de la Corte refiriéndose a un alto y poco realista estándar de prueba para determinar legalmente la complicidad de Serbia en genocidio. En este sentido, señaló que la exigencia de la Corte para probar que funcionarios serbios enviaron a Mladic instrucciones específicas para cometer genocidio, es una evidencia que obviamente nunca se encontraría. Insistiendo en este punto, Cassese cuestionó si no era suficiente demostrar que los mandos militares serbio-bosnios eran financiados y retribuidos por Serbia, además de existir una estrecha vinculación política entre ambos. ${ }^{20}$ En forma opuesta, Schabas opinó sobre Srebrenica, señalando que si bien las pruebas circunstanciales y otros medios de evidencia bastaron para establecer la culpabilidad del general Krstic, al mando allí de las tropas, ello difícilmente podría ser suficiente para vincularlo con las autoridades en Belgrado. ${ }^{21}$

La Corte tuvo la posibilidad de tener acceso a documentos clave en los que se establecen los vínculos entre autoridades serbias y serbio-bos-

cial intention como intención criminal agravada y al dolus specialis como dolo agravado. Cassese Antonio, op. cit., nota 6, pp. 103 y 167.

20 Véase a Antonio Cassese en The Guardian, Londres, 27 de febrero del 2007.

21 Schabas, William A., "First Judgments of the ICTY", Fordham International Law Journal, vol. 25, núm. 24, 2002, p. 53. 
nias. Se trata, entre otros, de las minutas del Consejo Serbio de Defensa (el órgano encargado del ejército yugoslavo); éstas fueron presentadas como evidencia en el caso Milosevic, pero, al parecer, Serbia obtuvo garantías del ICTY de no entregarlas a la Corte. ${ }^{22}$ Sólo así podemos explicarnos su negativa de pedir dichos documentos, no obstante la reiterada solicitud de la representación legal bosnia de una orden, demandando su entrega. En relación con estos documentos y al presunto "compromiso" de la Corte, el ex fiscal de Milosevic, Geoffrey Nice, en una carta enviada al diario Jutanji List de Zagrev, acusó a Carla del Ponte, fiscal general del ICTY, de haber hecho tratos con Belgrado para retener evidencia del juicio a Milosevic que pudiera ayudar en el caso de Bosnia contra Serbia. ${ }^{23}$ En respuesta, la Oficina de la Fiscalía General negó tal especie, aunque admitió el intercambio de correspondencia con Belgrado en una de cuyas misivas se dijo que no objetaría otorgar a ciertos documentos "medidas protectoras razonables".

22 Conviene aquí reproducir un párrafo de la opinión disidente del vice-presidente Khasawneh: "The Court should have required the Respondent to provide unedited copies of its Supreme Defense Council documents, failing which; the Court should have allowed a more liberal recourse to inference". Cabe señalar que en caso de que se produjera nueva evidencia sobre la responsabilidad de Serbia (por ejemplo, en futuros procesos del ICTY) podría solicitarse a la Corte la revisión del fallo de acuerdo con el artículo 61 de su Estatuto.

23 Véase International Herald Tribune del 16 de abril de 2007. En una entrevista reciente, Del Ponte confirmó que en mayo de 2003 envió una carta al canciller serbio, Goran Svilanovic, diciendo que aceptaría sellar algunas secciones "razonables" de los expedientes. "Ha sido una larga lucha para obtener los documentos y al final aceptamos debido a la presión del tiempo", señaló, y agregó que: "eran extremadamente valiosos para la convicción de Slobodan Milosevic". Antes, el 15 de febrero, Del Ponte hizo una importante declaración que implica una responsabilidad directa de Belgrado por genocidio: "You may wonder why my office is so keen to have access to the personnel file and full record for Ratko Mladic? Because the file can speak for itself and show that Ratko Mladic was not an outcast, not a lunatic who went on a rampage in Bosnia to fulfill his crazy ideas. No, he was a senior officer of the Yugoslav army on duty in Bosnia as commander of VRS; he was promoted twice during the war with highest marks in his dossier, the full approval of the Belgrade military and political leadership and with very generous remuneration If, for a moment, you combine this with the facts from the ground, known to the whole world, and reported to Belgrade - you have the fact established beyond any doubt - and that is that Belgrade was directly involved in the war in Bosnia. And later? Belgrade organized comfortable hiding places for Ratko Mladic in Serbia, so he can evade international justice. And where is he now? He is still in Serbia. Nothing was done to stop Mladic or his associates; nothing was done to punish him or his associates". 


\section{6. ¿Cómo determinó la Corte que el VRS no estaba bajo el control del SRJ?}

Respecto a la relación entre el ejército yugoslavo (VJ) y el ejército serbio bosnio (VRS), así como el apoyo económico de la República Federal de Yugoslavia (SRJ) a la Republika Sprska (RS), la Corte tomó nota de los argumentos de ambas partes (párrafos 236-240). Su conclusión fue: "The SRJ was making its considerable military and financial support available to the Republik Sprska, and had it withdrawn that support, this would have greatly constrained the options that were available to the Republik Sprska authorities" (párrafo 241).

Mucha de la incertidumbre sobre el veredicto ha girado en torno al por qué este hecho fue interpretado como una "falta en prevenir y sancionar" pero no como "complicidad". ${ }^{24}$ La conclusión paralela relativa a las fuerzas paramilitares (párrafos 385-395) puede arrojarnos alguna luz sobre esa contradicción, ya que para atribuir responsabilidad a un Estado por instancias no oficiales, la Corte requirió una demostración de su "total dependencia" (párrafo 392) del Estado. ${ }^{25}$ Este es un criterio alterno al que sólo requiere demostrar que el Estado ejerce un "control global" (párrafo 402), el cual la Corte rechazó por considerarlo demasiado amplio (párrafo 406). ${ }^{26}$ La principal razón para no determinar que los Scorpions

24 Cassese, op. cit., nota 6.

25 Esto significa que el control de un Estado sobre paramilitares u otros actores no estatales puede darse solamente si los individuos o grupos en cuestión actúan con "total dependencia" de dicho Estado, misma que la Corte no encontró en el VRS y las fuerzas paramilitares respecto de Serbia. Al aplicarse un umbral tan alto para determinar el control estatal, puede sentar un precedente que permita a los Estados llevar a cabo crímenes internacionales mediante actores no estatales sin que incurran en una responsabilidad directa. En esta situación, la Corte aplicó nuevamente el llamado estándar de "control efectivo" que la Corte había utilizado en el caso Nicaragua $v$. Estados Unidos. Véase Case Concerning Military and Paramilitary Actions in and Against Nicaragua (Nicaragua $v$. United States of America) en www.icj-cij.org/docket/index, y para un detallado análisis del caso, vease Laudi, Marion, Nicaragua ante la Corte Internacional de Justicia de La Haya, México, Siglo XXI, 1988.

26 La Corte debió haber utilizado el criterio aplicado por el ICYT en el caso Tadic, cuando descartó la prueba "Nicaragua" del "control efectivo". Así, sólo hubiera bastado con probar que el ejército yugoslavo ejerció un "control global” sobre el VRS —al planear, organizar y coordinar las operaciones - contrariamente a dar a las fuerzas las instrucciones específicas requeridas para probar una situación de control efectivo. Véase Schabas, op. cit., nota 8, pp. 243 y 244. 
fueran "totalmente dependientes", es que sus casos y los de Stanisic y Simatovic no hayan aún concluido (párrafo 395). Esta remisión a dichos casos en el ICTY es riesgosa y podría debilitar la determinación de que los actos de genocidio en Srebrenica no pueden atribuirse al demandado (párrafo 395). Sin embargo, la Corte determinó categóricamente como un hecho establecido la autoría del genocidio al VRS (párrafo 417). Respecto a si lo anterior exoneraba a la SRJ de complicidad en el genocidio, el razonamiento en el párrafo 422 nos da la respuesta:

Undoubtedly, the quite substantial aid of a political, military and financial nature provided by the FRY to the Republika Sprska abd the VRSm beginning long before the tragic events of Srebrenica, continued during those events. There is thus little doubt that the atrocities in Srebrenica were committed, at least in part, with the resources which the perpetrators of those acts possessed as a result of the general policy of aid and assistance pursued toward them by the FRY. However, the sole task of the Court is to establish the legal responsibility of the respondent, a responsibility which is subject to very specific conditions. One of those conditions is not fulfilled, because it is not established beyond any doubt in the arguments between the Parties whether the authorities of the FRY supplied - and continued to supply - the VRS leaders who decide upon and carried out those acts of genocide with their aid and assistance, at a time when those authorities were clearly aware that genocide was about to take place or was under way; in other words that not only ere massacres to be carried out or already under way, but that their perpetrators had the specific intent characterizing genocide, namely, the intent to destroy, in whole or in part, a human group, as such (párrafo 422).

En este caso, pareciera que la base para rechazar los cargos de complicidad es que el "pleno conocimiento" no había sido "concluyentemente establecido" (párrafo 423). ${ }^{27}$ Estimamos que nos encontramos aquí

27 Es interesante como veía Milanovic estas cuestiones un año antes del fallo de la Corte: "The issue of state responsibility of Serbia for the commission of genocide is factually a highly complex one, and will provide extensive probative difficulties to the Bosnian side. However, state responsibility of Serbia for complicity in genocide might be easier to prove, as the intense involvement in various paramilitary groups in the crimes of Bosnian war is much easier to attribute to Serbia... Serbia might also be held liable for direct and public incitement to genocide, though it would seem at first glance that most of the venom spewed by Belgrade's propaganda machinery did not reach the qualitative level (especially the directness) of the genocidal propaganda in Rwanda or Nazi Ger- 
frente a una disyunción al conectar el párrafo anterior con la determinación de la Corte de que las autoridades de la SRJ no podían haber sido ignorantes del grave riesgo de genocidio (párrafo 461).

Esto no aplicó al cargo de faltar en prevenir el genocidio. ${ }^{28}$ Ahí la Corte estableció: "The respondent has not shown that it took any initiative to prevent what happened, or any action on its part to avert the atrocities which were committed. It must therefore be concluded that the organs of the Respondent did nothing to prevent the Srebrenica massacres, claiming that they were powerless to do so, which hardly tallies with their own influence over the VRS" (párrafo 438).

De manera similar al cargo de falta en sancionar, la Corte admitió que las autoridades serbias, con conocimiento de causa, dieron refugio al menos a un fugitivo (párrafos 448-449).

\section{7. ¿Por qué determinó la Corte que el genocidio sólo se cometió en Srebrenica?}

En la larga sección (párrafos 246-277) en la que se plantearon otras conductas ilícitas fuera de Srebrenica, se determinó que éstas carecían de

many". Serbia certainly should be held responsible for failing to prevent genocide and failing to punish genocide, even if the "only" instance of genocide in Bosnia is the Srebrenica massacre... The distinction between the state obligation to prevent genocide and its duty not complicit in genocide under Article III of the Convention is somewhat difficult to draw, except by saying that complicity in genocide implies some sort of positive involvement in the commission of the crime, while failing to prevent genocide is an act of omission. Milanovic, Marko, op. cit., nota 12, p. 600.

28 Según recoge el diario Der Spiegel del 6 de junio del 2007, el pasado 4 de junio familiares de los victimados en Srebrenica presentaron en La Haya, a través de sus abogados, una demanda civil ante la Corte Suprema de Holanda contra el gobierno de ese país y las Naciones Unidas por no haber protegido adecuadamente a la población. Ya antes, el desastroso papel desempeñado por el destacamento holandés de Unprofor fue investigado por una comisión gubernamental que incluso llevó a la dimisión del primer ministro Wim Kok en 2002. Esta vez se está yendo más lejos; no sólo se trata de la responsabilidad estatal por genocidio, sino de las propias Naciones Unidas. Habrá que seguir con mucha atención este proceso legal en que se acusa a los soldados holandeses de negligencia criminal al faltar a su deber de salvaguardar la zona protegida de la ONU, toda vez que "entregaron a la población a los serbio-bosnios sedientos de sangre". Además de ello, estimamos que la Unprofor y el propio Consejo de Seguridad, creación del mismo, pueden incurrir en una actuación ultra vires. En este sentido, véase Portilla Gómez, Juan Manuel, "Naturaleza jurídica y contenido actual de las sanciones económicas. El caso de Irak", Anuario Mexicano de Derecho Internacional, UNAM, Instituto de Investigaciones Jurídicas, vol. V, 2005, p. 402. 
pruebas o que constituían algo diferente al genocidio. El punto central de su razonamiento se relaciona con la "intención específica" (párrafo 277), aunque la Corte explícitamente señala el hecho de que otros elementos de genocidio, particularmente el asesinato de un número significativo de miembros de un grupo, estuvieron presentes. Una determinación similar se hizo respecto a las violaciones sistemáticas y a otros abusos físicos: se establecieron los hechos, pero no la intención (párrafo 334). Y nuevamente la misma determinación se hizo, referente al sistemático bombardeo y los ataques de los francotiradores en contra de las poblaciones urbanas: se establecieron los hechos, pero no la intención (párrafo 328). Y otra vez la misma determinación se hizo respecto a la expulsión y el reasentamiento forzado: se establecen los hechos, pero no la intención (párrafo 334). Y nuevamente la misma determinación se hace con relación al trato de los prisioneros en los campos de detención: se establecen los hechos, pero no la intención (párrafo 354). Específicamente rechazaron los argumentos sobre un punto, el cual ocupó una gran parte del ángulo mediático de la guerra: el uso de la violación y la violencia sexual como un medio forzado para producir alteraciones demográficas (párrafos $355-367) .{ }^{29}$

Al considerar la destrucción de propiedad cultural y religiosa, la Corte determinó que la evidencia establecía los hechos, pero decidió no considerar la destrucción de esa índole como genocidio (párrafo 344). Debido a su determinación de interpretar restrictivamente la Convención de

29 Bosnia alegó que la separación de hombres y mujeres, en combinación con los efectos de la violación de éstas, aunada a la mutilación genital de los hombres, produjeron alteraciones demográficas en tanto que incidieron en la reducción de nacimientos de bebés en el seno del grupo protegido (en contravención del artículo II de la Convención de Genocidio). De igual forma, se argumentó también que al procrear las mujeres musulmanas a pequeños chetniks se alteraba la composición demográfica. Conviene aquí precisar que la violación y otros actos de agresión sexual constituyen crímenes contra la humanidad (juzgados por el ICTY y otros tribunales internacionales). En relación con cuestiones de género, es interesante observar que en Bosnia se cometió genocidio en contra de la población masculina: más de un $90 \%$ de los asesinados fueron hombres. Respecto a la violación de mujeres, véanse Dawn Askin, Nelly, War Crimes Against Women: Prosecution in International War Crimes Tribunals, La Haya, Martinus Nijhoff, 1997, y Tétreault, Mary Ann, “Justice for All: Wartime Rape and Women's Human Rights”, Global Governance, vol. 3, núm. 3, 1997, 197-212; por cuanto al genocidio por género, véase "Case Study: Bosnia Herzegovina", Gendercide Watch, www.gendercide.org/case bosnia.html. 
Genocidio, no sorprende que la Corte haya decidido no sentar un precedente que expandiera la definición de genocidio en ese sentido. Pero su planteamiento en los hechos y su inclusión en el expediente no está exento de importancia En efecto, la Corte suscribió la determinación del ICYT de que: "Where there is physical and biological destruction there are often simultaneous attacks on the cultural and religious property and symbols of the targeted group as well, attacks which may legitimately be considered as evidence of intent to physically destroy the group" (párrafo 344$).{ }^{30}$

Aún mayor resultó el apego de la Corte a las conclusiones sobre Srebrenica del ICTY, al determinar que ahí se cometió genocidio con base en sus decisiones en los casos Krstic (párrafos 292-293) y Blagojevic (párrafos 294-295). ${ }^{31}$ Sin embargo, hasta ahí llegó la Corte al no salirse de Sebrenica ni del lapso comprendido entre el 13 y el 18 de julio de 1995. ${ }^{32}$ En tan corto tiempo se masacraron a ocho mil hombres y mucha-

30 Creemos que de este modo se abrió la puerta para considerar en lo futuro la destrucción cultural como evidencia de intención genocida, independientemente de que la Corte haya decidido no hacerlo ahora.

31 A escasos tres meses del fallo de la Corte, el 9 de mayo del 2007, la Sala de Apelaciones del ICYT revirtió el cargo de genocidio que la Sala de Primera instancia había fincado a Vidoje Blagoyevic, Case No. IT-02-60-A. Esto no es un asunto menor, ya que se suma a otros 13 en los que no se llegó a la sentencia por tal crimen (incluido el de Milosevic) y sólo queda Radoslav Kristic como el único convicto por genocidio. Se llegó a tal conclusión al considerarlo ajeno a la intención genocida de los principales perpetradores de la masacre de Srebrenica, y por lo tanto no se le encontró culpable de complicidad en genocidio, aunque se ratificaron otros cargos por su papel en dichos hechos. Su sentencia se redujo de 18 a 15 años de prisión. Actualmente están en proceso siete altos mandos militares y policiales serbio-bosnios, y cinco de ellos — Ljubisa Beara, Ljubomir Borovcanin, Vujadin Popovic, Drago Nikolic y Vinko Panderevic - han sido acusados de genocidio. Sin embargo, el panorama no es muy halagüeño luego de la exigencia de un estándar de prueba tan alto de la Corte, y ahora también de la Sala de Apelaciones del ICTY (¡que significó un cambio de 360 grados sobre la previa decisión de la Sala de Primera Instancia!), sobre todo, considerando que la Corte se basó en este caso, junto con el de Kristic, para establecer el genocidio en Srebrenica. Por ello será muy difícil probar estos cargos satisfactoriamente, y que los jueces que evalúen la evidencia (la cual será muy similar a la presentada en los casos anteriores) se convenzan de que los acusados compartieron la intención genocida con los arquitectos del genocidio Ratko Madlic, comandante del ejército sebio-bosnio, y el líder serbio-bosnio Radovan Karadzic. Para consultar los procesos del ICTY, véase www.un.org/icty.

32 En un artículo publicado en el International Herald Tribune del 8 de marzo de 2007, Ruth Wedgwood opinó que al decir que sólo en Srebrenica se cometió genocidio, 
chos que vinieron a sumarse a la muerte en toda Bosnia de 150 mil civiles, de 1992 a 1995, en actos igualmente constitutivos de genocidio. Más aún, de acuerdo con lo que arrojó el caso Milosevic, existen importantes elementos para considerar que el genocidio se cometió también en otras localidades, así la Sala de Primera Instancia del ICTY determinó, el 16 de junio del 2004, que había "suficiente evidencia de que se cometió genocidio en Brcko, Prijedor, Snaski Most, Srebrenica, Bijeljina, Kijuc y Bosanski Novi". ${ }^{33}$ Por su parte, Wedgwood nos da otra pista al preguntar en qué se diferencia la deliberada matanza de civiles en el pueblo ribereño de Brcko en 1992, para desalojar a los musulmanes del corredor del río Sava, o la tortura y la inanición de civiles musulmanes en Foca, a las masacres de Srebrenica, que buscaban resguardar el valle de Drina. ${ }^{34}$

\section{REFLEXIONES FINALES}

A lo largo de su historia, Bosnia ha estado atrapada entre imperios, $\mathrm{y}$ tras la disolución de Yugoslavia se despertaron los fantasmas del pasado combinándose con una renovada versión nacionalista que muy pronto desplazó a la ideología comunista. La violencia desatada tuvo como base la división étnica, y paradójicamente el término de la misma se basó igualmente en la división étnica representada en el Acuerdo de Dayton, en el que cual se dotó de legalidad a la entidad agresora, la auto-denominada Republika Sprska, dentro de una federación con los croatas y los musulmanes bosnios.

No obstante la experiencia traumática y devastadora en la Alemania nazi sufrida por la población judía y otros grupos étnicos, la Carta de las Naciones Unidas, en apego estricto al principio de no intervención en los asuntos internos de los Estados, confinó las violaciones masivas a los de-

la Corte limita los cargos que puedan efectivamente hacerse en contra de los líderes serbio-bosnios Radovan Karadzic y Ratko Mladic, siempre y cuando Belgrado permita su arresto.

33 Case No. IT-02-54-T, www.un.org/icty. Slobodan Milosevic, presidente de Serbia, fue el primer caso de un jefe de Estado juzgado por un tribunal penal internacional. Tras la caída de su gobierno en octubre del 2000, fue detenido en abril del 2001 y entregado dos meses después al ICTY. Se le abrió un proceso por más de 60 cargos, y meses antes de que se le dictara sentencia, falleció el 11 de febrero del 2006 en su celda en La Haya.

34 Wedgwood, R., op. cit., nota 32. 
rechos humanos dentro de las fronteras de los mismos. Este enfoque comenzó a subsanarse a partir de la Declaración Universal de Derechos Humanos y de la propia Convención de Genocidio, que fueron adoptadas casi simultáneamente por Asamblea General en diciembre de 1948.

Los crímenes contra el derecho internacional son cometidos por personas con nombre y apellidos, no por entidades abstractas, y sólo al sancionar a los individuos perpetradores de tales crímenes pueden hacerse cumplir las disposiciones del derecho internacional.

A partir de Nuremberg, el desarrollo del derecho penal internacional ha reforzado dicho criterio. El surgimiento del régimen de los derechos humanos y de los Convenios de Ginebra ha convertido a los individuos en legítimos agentes del sistema internacional. Con la creación del ICTY, del ICTR y de la Corte Penal Internacional la responsabilidad de los individuos ha quedado subsumida dentro del sistema de justicia penal internacional.

De acuerdo con este régimen, los individuos pueden ser acusados de genocidio y otros crímenes internacionales. Sin embargo, cuando dichos individuos oprimen el gatillo, violan y masacran, no lo hacen a título personal. Actúan dentro y/o a través de las estructuras oficiales, como agentes de las mismas, ya sean de jure o de facto. Por ello, cuando los individuos han sido acusados y sancionados legalmente por la comisión de estos crímenes, es del todo apropiado que los Estados sean encontrados también responsables, debido a que sólo una comunidad organizada cuenta con los medios necesarios para infligir violencia a tan grande escala.

Lo anterior no quiere decir que debamos sancionar solamente a los Estados: los individuos deben ser responsables y sancionados por las violaciones al derecho internacional. Nuestro argumento va más bien en el sentido de que los individuos deben hacerse responsables en paralelo con los Estados, y que el sistema debe funcionar de tal modo que la sanción de unos no sea excluyente de la sanción del otro.

La Convención de Genocidio tiene una inadecuada cobertura. Por ejemplo, los grupos políticos y económicos fueron omitidos; se excluye el genocidio cultural sin considerar que la privación del lenguaje, ritos y religión puede ser tan efectiva en la aniquilación de un grupo como su exterminio. De hecho, la transferencia de niños, la cual está condenada por la Convención, constituye más un ataque cultural que biológico en la integridad de un grupo. Asimismo, la Convención es ambigua en la defi- 
nición de los criterios para determinar si un grupo ha sido destruido "en parte”. ¿Qué sucede si un género o segmento del grupo sólo es afectado? ¿Si únicamente las víctimas son hombres o mujeres, campesinos o intelectuales?

La incapacidad de la Corte para determinar la responsabilidad directa de Serbia, o al menos su complicidad en la autoría del genocidio se debió a también a que aplicó las exigencias de un proceso penal a un caso de responsabilidad civil. De ahí la imposibilidad del equipo legal bosnio de aportar pruebas completamente conclusivas e incontrovertibles requeridas por la Corte. En efecto, el ámbito propio de la Corte no es precisamente el derecho penal: ésta se encuentra constreñida por cuestiones de soberanía e intereses de los Estados, a veces más que de justicia, y fue más que obvio lo forzado de su papel al moverse en aguas que le son ajenas.

Pero aún dentro de un tribunal penal como el ICTY, el asunto del genocidio no es tarea fácil como lo demuestra el alto número de casos en que dicho cargo no ha procedido. En el más reciente y desalentador de ellos, Vidoje Blagojevic fue absuelto por la Sala de Apelaciones, revirtiendo así lo que se esperaba sólo como una confirmación de lo determinado anteriormente por la Sala de Primera Instancia. Fueron los mismos hechos, la misma evidencia, los mismos testimonios y las mismas opiniones de expertos. Lo único distinto fue la sentencia. Resulta difícil entender cómo pudieron pensar tan diferente los jueces de apelación de los de primera instancia, y podemos pensar que anda algo anda mal en el sistema. Creemos que esto envía un mensaje confuso sobre el procesamiento del genocidio, y más aún que este caso fue uno de los dos en que la Corte se apoyó para determinar la comisión del genocidio.

¿Justicia en los Balcanes? Muy poca, un proceso que duró casi tres lustros sin aportar ningún alivio a las víctimas y que negó la responsabilidad estatal, nada abona a quienes esperábamos que la Corte asumiera un papel más proactivo en el combate a la impunidad en la comisión del más grave crimen internacional. Creemos que todo ello no augura buenas expectativas para Croacia en su demanda contra Serbia, y que el fallo de la Corte, lejos de constituir un factor disuasivo, alentará a los regímenes perpetradores de genocidio. 
nición de los criterios para determinar si un grupo ha sido destruido "en parte”. ¿Qué sucede si un género o segmento del grupo sólo es afectado? ¿Si únicamente las víctimas son hombres o mujeres, campesinos o intelectuales?

La incapacidad de la Corte para determinar la responsabilidad directa de Serbia, o al menos su complicidad en la autoría del genocidio se debió a también a que aplicó las exigencias de un proceso penal a un caso de responsabilidad civil. De ahí la imposibilidad del equipo legal bosnio de aportar pruebas completamente conclusivas e incontrovertibles requeridas por la Corte. En efecto, el ámbito propio de la Corte no es precisamente el derecho penal: ésta se encuentra constreñida por cuestiones de soberanía e intereses de los Estados, a veces más que de justicia, y fue más que obvio lo forzado de su papel al moverse en aguas que le son ajenas.

Pero aún dentro de un tribunal penal como el ICTY, el asunto del genocidio no es tarea fácil como lo demuestra el alto número de casos en que dicho cargo no ha procedido. En el más reciente y desalentador de ellos, Vidoje Blagojevic fue absuelto por la Sala de Apelaciones, revirtiendo así lo que se esperaba sólo como una confirmación de lo determinado anteriormente por la Sala de Primera Instancia. Fueron los mismos hechos, la misma evidencia, los mismos testimonios y las mismas opiniones de expertos. Lo único distinto fue la sentencia. Resulta difícil entender cómo pudieron pensar tan diferente los jueces de apelación de los de primera instancia, y podemos pensar que anda algo anda mal en el sistema. Creemos que esto envía un mensaje confuso sobre el procesamiento del genocidio, y más aún que este caso fue uno de los dos en que la Corte se apoyó para determinar la comisión del genocidio.

¿Justicia en los Balcanes? Muy poca, un proceso que duró casi tres lustros sin aportar ningún alivio a las víctimas y que negó la responsabilidad estatal, nada abona a quienes esperábamos que la Corte asumiera un papel más proactivo en el combate a la impunidad en la comisión del más grave crimen internacional. Creemos que todo ello no augura buenas expectativas para Croacia en su demanda contra Serbia, y que el fallo de la Corte, lejos de constituir un factor disuasivo, alentará a los regímenes perpetradores de genocidio. 